\title{
AGGREGATIVE MOVEMENT AND FRONT PROPAGATION FOR BI-STABLE POPULATION MODELS
}

\author{
PHILIP K. MAINI \\ Centre for Mathematical Biology, Mathematical Institute, \\ University of Oxford, OX1 3LB Oxford, UK \\ maini@maths.ox.ac.uk \\ LUISA MALAGUTI \\ Department of Engineering Sciences and Methods, \\ University of Modena and Reggio Emilia, \\ I-42100 Reggio Emilia, Italy \\ malaguti.luisa@unimore.it \\ CRISTINA MARCELLI \\ Department of Mathematical Sciences, \\ Polytechnic University of Marche, \\ I-60131 Ancona, Italy \\ marcelli@dipmat.univpm.it \\ SERENA MATUCCI \\ Department of Electronic and Telecommunications, \\ University of Florence, I-50139 Florence, Italy \\ serena.matucci@unifi.it \\ Received 28 August 2006 \\ Revised 15 December 2006 \\ Communicated by N. Bellomo
}

Front propagation for the aggregation-diffusion-reaction equation

$$
v_{\tau}=\left[D(v) v_{x}\right]_{x}+f(v)
$$

is investigated, where $f$ is a bi-stable reaction-term and $D(v)$ is a diffusion coefficient with changing sign, modeling aggregating-diffusing processes. We provide necessary and sufficient conditions for the existence of traveling wave solutions and classify them according to how or if they attain their equilibria at finite times. We also show that the dynamics can exhibit the phenomena of finite speed of propagation and/or finite speed of saturation.

Keywords: Diffusion-aggregation models; population dynamics; traveling wave solutions; finite speed of propagation.

AMS Subject Classification: 35K25, 92D25, 34B40, 34B16 


\section{Introduction}

Reaction-diffusion equations of the form

$$
v_{\tau}=(\Phi(v))_{x x}+f(v), \quad x \in \mathbb{R}, \quad \tau \geq 0
$$

with $\Phi(0)=0, \Phi$ monotone increasing, have been widely used in the literature (see, for instance, Ref. 12 and the references therein) in order to describe the spatial dispersion of a population. In (1.1) $v(x, \tau)$ denotes the population density at time $\tau$ and location $x$; the reaction term $f(v)$ accounts for the rate at which individuals enter the population due to births and deaths, while the diffusion term $\Phi(v)$ represents the dispersal of the population. In writing (1.1) it is assumed that births and deaths do not depend on time and location.

Equation (1.1) with $\Phi$ increasing cannot model frequently observed phenomena such as swarming or local grouping of individuals since it describes the case in which all the individuals migrate toward regions of lower density.

Several authors have recently proposed various mathematical models aimed at describing the phenomenon of aggregation of populations. ${ }^{3,7,13,15}$ In most cases the function $\Phi$ is assumed to be monotone increasing in $(0, \beta)$ and monotone decreasing in $(\beta, 1), \beta$ being a suitable critical value of the density $v$. In this way the term $\Phi$ takes into account both the dispersal of individuals due to random motion and the tendency of the population to crowd. When the density is small, dispersal prevails, while for population densities beyond the critical value $\beta$ the tendency to aggregate becomes predominant. This paper is devoted to the investigation of Eq. (1.1) under this condition of aggregation-diffusion on the term $\Phi$.

Particular cases of (1.1) have been derived and partially analyzed in Refs. 7, 9, 13 and 15, but several open problems remain. Indeed, the initial-boundary value problem associated with (1.1) can be ill-posed ${ }^{1,2}$; however, in Ref. 13 existence and uniqueness of a global solution are shown for the perturbed equation, obtained from (1.1) by adding a regularizing term $\left(\lambda v_{t}-\lambda f(v)\right)_{x x}$ with $\lambda>0$. It is worth remarking that the discrete model underlying (1.1) is well-posed, ${ }^{7,15}$ and numerical computations show a good agreement between the information obtained in the discrete setting and the predictions derived from (1.1). We refer the reader interested in the problem of well-posedness of (1.1) to the discussion presented in Ref. 9 and to the references therein.

Existence and properties of traveling wave solutions (denoted t.w.s. from now on) between the stationary states 0 and 1, for Eq. (1.1) with an aggregation-diffusion term $\Phi$ and a Fisher-KPP (monostable) reaction term $f$, i.e. satisfying $f(v)>0$ in $(0,1), f(0)=f(1)=0$, have been analyzed by the authors in Ref. 9. T.w.s. play a relevant role in the study of reaction-diffusion equations, since in some cases ${ }^{4,8}$ they attract solutions whose initial datum is not too far from the wave profile. In our context of possible ill-posedness of the initial value problem for Eq. (1.1), t.w.s. are regular solutions whose profile seems in good agreement with the numerical investigations done in Ref. 13 for the perturbed dynamic. The existence of a threshold value $c^{*}$ was proved in Ref. 9 such that (1.1) supports t.w.s. with speed $c$ if and 


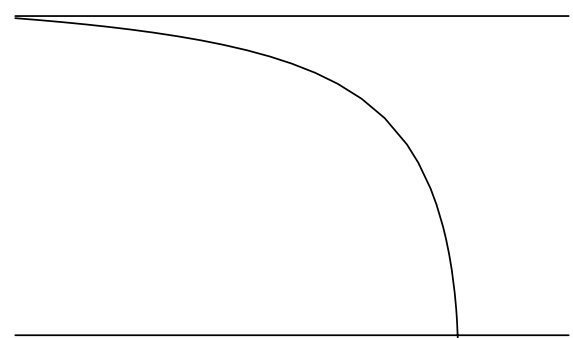

sharp of type (I) t.w.s.

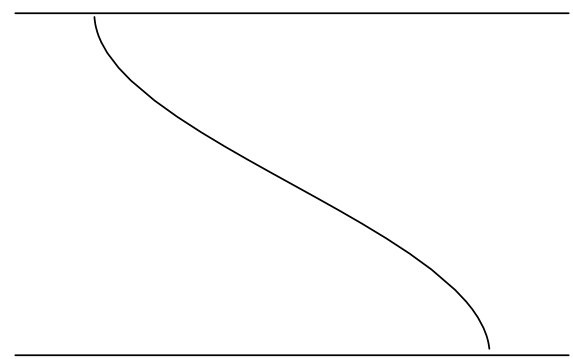

sharp of type (III) t.w.s.

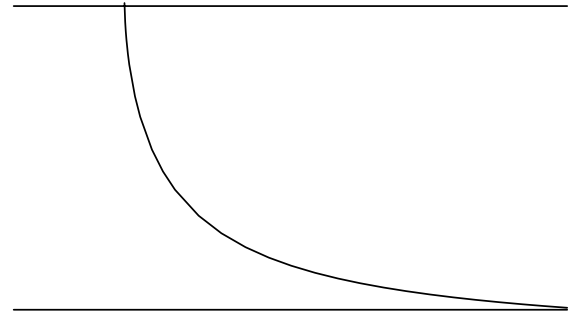

sharp of type (II) t.w.s.

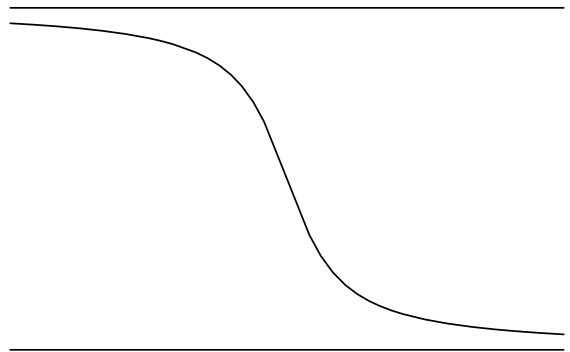

front-type t.w.s.

Fig. 1. Possible t.w.s. solutions for Eq. (1.1) with critical wavespeed (see text for details).

only if $c \geq c^{*}$. The properties of such fronts have also been investigated. Indeed, the possible degeneracy of the diffusion-aggregation term (occurring when $\dot{\Phi}(0)=0$ and/or $\dot{\Phi}(1)=0$ ) causes the appearance of fronts which attain the equilibria 0 and/or 1 at finite times. When $c>c^{*}$ the dynamics admit only classical (i.e. fronttype) t.w.s., instead, for $c=c^{*}$ one of the four possible types of t.w.s. illustrated in Fig. 1 appears and we refer to the following section for their definition.

We underline that reaching the equilibrium 1 at a finite time is typical of diffusion-aggregation processes, since this does not occur in the case of reactiondiffusion equations (with positive diffusion), even if $\dot{\Phi}(1)=0 .{ }^{10}$ Dynamics exhibiting such phenomena are generally said to have the properties of finite speed of saturation (when the t.w.s. attains the equilibrium 1 at a finite time), and/or finite speed of propagation (when the t.w.s. reaches the equilibrium 0 at a finite time). In the context of merely diffusive processes, Gilding and Kersner ${ }^{5}$ proved that when a right-compact profile appears (i.e. a t.w.s. of sharp type (I) or (III), see Fig. 1), then all the solutions $v(x, \tau)$ of $(1.1)$, with initial condition $v(x, 0)$ having compact support, maintain a uniformly bounded support for all $\tau$ in a right neighborhood of 0 . In other words, a population initially localized in an arbitrarily bounded region diffuses in the habitat with finite speed. Due to this property, degenerate reactiondiffusion equations are appropriate models for biological diffusion. It is open to question whether the appearance of right-compact supported t.w.s. induces the same property in aggregation processes (1.1). 
The aim of this paper is to analyze front propagation when the reproduction rate satisfies the Allee effect, ${ }^{13}$ that is when there is a critical value $\alpha$ of the population density such that below it the death rate is higher than the birth rate, while above it the opposite situation occurs. Formally, this means that $f$ is a bi-stable reaction term satisfying

$$
f(0)=f(\alpha)=f(1)=0, \quad f(v)<0 \text { in }(0, \alpha), \quad f(v)>0 \text { in }(\alpha, 1)
$$

for some $\alpha \in(0,1)$. Jointly with Ref. 9 , this investigation begins with a theory on the existence of t.w.s. and illustrates their properties in diffusion-aggregation processes. What is relevant in the present case (i.e. when $f(u)$ satisfies (1.2)), is that the existence of wavefront connections between the stationary states 0 and 1 is no longer guaranteed, but depends on the relative behavior of $\alpha$ and $\beta$. Indeed (see Theorem 2.1), necessary conditions for the existence of t.w.s. are

$$
\alpha<\beta \quad \text { and } \quad \int_{0}^{\beta} f(u) \dot{\Phi}(u) d u>0 .
$$

Moreover, such conditions are not sufficient since the existence of t.w.s. connecting 1 to 0 is also linked, in some sense, to the existence of heteroclinics connecting 1 to $\beta$ and $\beta$ to 1 . This is shown in Theorem 3.1, where we also prove that the admissible wave speed is unique and we give a criterion to classify the resulting t.w.s. into one of the four types described above.

As is natural, the necessary and sufficient condition stated in Theorem 3.1 has an implicit form, since it involves the wave speeds between 0 and $\beta$ and between $\beta$ and 1. So, in Theorem 3.2 we give some simple explicit conditions ensuring the validity or not of the necessary and sufficient condition of Theorem 3.1, and then the existence or non-existence of t.w.s. An example completes the discussion.

The main tools used for our investigation are based on upper-lower solutions and comparison techniques in the phase plane. Indeed, due to the mild regularity assumptions on the coefficients, the usual methods from dynamical systems theory cannot be applied in this context.

\section{Preliminaries and Necessary Conditions}

As usual, a t.w.s. for Eq. (1.1) is a solution having the form $v(\tau, x)=u(x-c \tau)$ for some constant $c$. The wave profile $u$ is a solution of the equation

$$
\left(D(u) u^{\prime}\right)^{\prime}+c u^{\prime}+f(u)=0, \quad 0 \leq u \leq 1
$$

where $\left({ }^{\prime}\right)$ stands for derivation with respect to the wave coordinate $t=x-c \tau$, $D(u):=\dot{\Phi}(u) \in C^{1}([0,1])$. As illustrated in the Introduction, our study concerns dynamics for which $D(u)$ satisfies

$$
(u-\beta) D(u)<0 \quad \text { in }(0,1) \backslash\{\beta\}
$$

for some given $\beta \in(0,1)$, and $f \in C[0,1]$ satisfies (1.2) with $\alpha \in(0,1)$. 
A function $u$ is a solution of (E) in its maximal existence interval $I=(a, b) \subseteq \mathbb{R}$ if $0 \leq u(t) \leq 1$ in $I, u \in C^{1}(I), D(u) u^{\prime} \in C^{1}(I)$, and $u$ satisfies (E) in $I$. We are interested in the existence of solutions of (E) satisfying the boundary conditions

$$
\begin{gathered}
u\left(a^{+}\right)=1, \quad u\left(b^{-}\right)=0 \\
\lim _{t \rightarrow a^{+}} D(u(t)) u^{\prime}(t)=\lim _{t \rightarrow b^{-}} D(u(t)) u^{\prime}(t)=0 .
\end{gathered}
$$

We start our investigation by analyzing the properties of the possible solutions $u$ of Eq. (E) satisfying (2.2) and (2.3).

Proposition 2.1. Let $u$ be a solution of (E) satisfying (2.2). Then

$$
\begin{aligned}
& a=-\infty \quad \text { implies } \lim _{t \rightarrow-\infty} D(u(t)) u^{\prime}(t)=0, \\
& b=+\infty \text { implies } \lim _{t \rightarrow+\infty} D(u(t)) u^{\prime}(t)=0 .
\end{aligned}
$$

Proof. Assume, for instance, $a=-\infty$. Let $T_{1}:=\sup \{t: u(\tau)>M=\max \{\alpha, \beta\}$ for every $\tau \in(-\infty, t)\}$ and define the functions

$$
G(u):=\int_{M}^{u} D(s) f(s) d s, \quad \Sigma(t):=\frac{1}{2}\left[D(u(t)) u^{\prime}(t)\right]^{2}+G(u(t)),
$$

for $u \in(M, 1), t \in\left(-\infty, T_{1}\right)$. Since $u$ satisfies $(\mathrm{E})$, it follows that

$$
\frac{d}{d t} \Sigma(t)=-c D(u(t))\left(u^{\prime}(t)\right)^{2}
$$

which has the same sign as $c$, when $c \neq 0$, since $u(t)>M$ in $\left(-\infty, T_{1}\right)$ and then $D(u(t))<0$. Hence $\Sigma(t)$ is monotone for $t<T_{1}$ and $\lim _{t \rightarrow-\infty} \Sigma(u(t))$ exists, being either finite or infinite. Since

$$
\lim _{t \rightarrow-\infty} G(u(t))=\int_{M}^{1} D(s) f(s) d s \in \mathbb{R}
$$

the existence of $\lim _{t \rightarrow-\infty} D(u(t))\left|u^{\prime}(t)\right|=: \ell \in[-\infty, 0]$ follows. If $\ell<0$, taking into account that $u(-\infty)=1, D(1) \leq 0$, we obtain the existence of $\lim _{t \rightarrow-\infty}\left|u^{\prime}(t)\right|=: \ell_{1}>0$ (possibly $\ell_{1}=+\infty$ ) which is impossible since $u$ is bounded. Therefore $\ell=0$ and the statement follows.

The case $b=+\infty$ can be treated by means of an analogous argument.

In view of the previous proposition, if $u$ is a solution of (E) and (2.2) such that $a=-\infty \quad(b=+\infty)$, then the corresponding condition in (2.3) is automatically satisfied. On the other hand, if $u$ is a solution of (E) satisfying (2.2)-(2.3) and such that $a>-\infty[b<+\infty]$, then $\lim _{t \rightarrow a^{+}} u^{\prime}(t) \neq 0\left[\lim _{t \rightarrow b^{-}} u^{\prime}(t) \neq 0\right]$. Indeed, if $u^{\prime}\left(a^{+}\right)=0\left[u^{\prime}\left(b^{-}\right)=0\right]$, then the function which is equal to $u$ in $I$ and identically 1 in $(-\infty, a]$ [identically 0 in $[b,+\infty)]$ is still a solution of $(\mathrm{E})$, in contradiction with the definition of $I$ as maximal existence interval.

If $I=\mathbb{R}$ the solution $u$ is called a front-type solution, or classical solution. Notice that, as an immediate consequence of Proposition 2.1, the present definition reduces 
to the usual one,${ }^{10,14}$ since in this case both the conditions in (2.3) are implied by (E) and (2.2). However, in our present general setting, due to the possible degeneracy of Eq. (E) at the equilibria (when $D(0)=0$ and/or $D(1)=0$ ), other types of solutions can appear, attaining the equilibria at finite values with negative (possibly infinite) slope; we will call them sharp solutions, distinguishing three different types depending on the existence interval. If $I=(-\infty, b)$, the solution $u$ is called sharp of type (I) and it is right compactly supported. If $I=(a, \infty)$, the solution $u$ is called sharp of type (II) and it is left compactly supported. If $I=(a, b)$ the solution $u$ is called sharp of type (III) and it is compactly supported (see Fig. 1). Throughout the paper $(a, b)$ will always denote the maximal existence interval of a solution $u(t)$ of Eq. (E).

The following result provides necessary conditions for the solvability of problem (E), (2.2)-(2.3).

Theorem 2.1. If Eq. (E) admits solutions satisfying conditions (2.2)-(2.3), then

$$
\alpha<\beta, \quad c>0, \quad \int_{0}^{\beta} f(u) D(u) d u>0 .
$$

Moreover, if $u(t)$ is a solution, then $u^{\prime}(t)<0$ whenever $0<u(t)<1$.

Proof. The proof will proceed by steps. Let

$$
\begin{aligned}
& T_{1}:=\sup \{t \in(a, b): u(\tau)>\max \{\alpha, \beta\} \text { for every } \tau \in(a, t)\}, \\
& T_{2}:=\inf \{t \in(a, b): u(\tau)<\min \{\alpha, \beta\} \text { for every } \tau \in(t, b)\}, \\
& \tau_{1}:=\inf \left\{t \in\left(a, T_{1}\right): u(\tau)<1 \text { for every } \tau \in\left(t, T_{1}\right]\right\}, \\
& \tau_{2}:=\sup \left\{t \in\left(T_{2}, b\right): u(\tau)>0 \text { for every } \tau \in\left[T_{2}, t\right)\right\} .
\end{aligned}
$$

Of course, $u\left(T_{1}\right)=\max \{\alpha, \beta\}, u\left(\tau_{1}^{+}\right)=1, u\left(T_{2}\right)=\min \{\alpha, \beta\}, u\left(\tau_{2}^{-}\right)=0$, $u^{\prime}\left(T_{1}\right) \leq 0, u^{\prime}\left(T_{2}\right) \leq 0$.

Step 1. If $u$ is a solution of (E) satisfying (2.2)-(2.3), then

(i) $u^{\prime}(t)<0$ in $\left(\tau_{1}, T_{1}\right) \cup\left(T_{2}, \tau_{2}\right)$;

(ii) if $\tau_{1}>a$ then $a=-\infty$ and $u(t) \equiv 1$ in $\left(-\infty, \tau_{1}\right)$;

(iii) if $\tau_{2}<b$ then $b=+\infty$ and $u(t) \equiv 0$ in $\left(\tau_{2},+\infty\right)$.

First let us prove that $u^{\prime}(t) \leq 0$ whenever $t \in\left(a, T_{1}\right) \cup\left(T_{2}, b\right)$. To this aim, assume by contradiction that $u^{\prime}\left(t^{*}\right)>0$ for some $t^{*} \in\left(T_{2}, b\right)$. Since $u\left(b^{-}\right)=0$, we deduce the existence of a value $t_{0}>t^{*}$ such that $u^{\prime}\left(t_{0}\right)=0$ and $u^{\prime}(t)>0$ in $\left(t^{*}, t_{0}\right)$. Of course, we have $0<u\left(t_{0}\right)<\alpha$, so put $h(t):=D(u(t)) u^{\prime}(t)$. By (E) we have $h^{\prime}\left(t_{0}\right)=-f\left(u\left(t_{0}\right)\right)>0$, i.e. $h(t)$ is increasing in a neighborhood of $t_{0}$, with $h\left(t_{0}\right)=0$. Since $D\left(u\left(t_{0}\right)\right)>0$ we have that $u^{\prime}(t)<0$ in a left neighborhood of $t_{0}$, in contradiction with the definition of $t_{0}$, so $u^{\prime}(t) \leq 0$ for every $t \in\left(T_{2}, b\right)$.

Let us now assume, again by contradiction, that $u^{\prime}\left(\tau^{*}\right)>0$ for some $\tau^{*} \in$ $\left(a, T_{1}\right)$. Put $\tau_{0}:=\sup \left\{t: u^{\prime}(\tau)>0\right.$ for every $\left.\tau \in\left(\tau^{*}, t\right)\right\}$. Of course, $\tau_{0}<T_{1}$ and 
$u^{\prime}\left(\tau_{0}\right)=0$. Note that $u\left(\tau_{0}\right)<1$. Indeed if $u\left(\tau_{0}\right)=1$, then integrating (E) in $\left(t, \tau_{0}\right)$ and taking into account that $f(u)>0$ for $u \in(\alpha, 1)$, we obtain that there exists the limit $\lim _{t \rightarrow a^{+}} D(u(t)) u^{\prime}(t)=\int_{a}^{\tau_{0}} f(u(t)) d t>0$, in contradiction with condition (2.3). So, $\beta<u\left(\tau_{0}\right)<1$ and then $h^{\prime}\left(\tau_{0}\right)=-f\left(u\left(\tau_{0}\right)\right)<0$, i.e. $h(t)$ is decreasing in a neighborhood of $\tau_{0}$, with $h\left(\tau_{0}\right)=0$. Since $D\left(u\left(\tau_{0}\right)\right)<0$, this means that $u^{\prime}(t)<0$ in a left neighborhood of $\tau_{0}$, in contradiction with the definition of $\tau_{0}$. Hence, $u^{\prime}(t) \leq 0$ for every $t \in\left(a, T_{1}\right)$.

By the same argument used above, it is easy to prove that if $u^{\prime}\left(t_{0}\right)=0$ for some $t_{0} \in\left(\tau_{1}, T_{1}\right) \cup\left(T_{2}, \tau_{2}\right)$, then $u^{\prime}(t)>0$ in a right neighborhood of $t_{0}$, in contradiction with what was proved above, and (i) follows. Now, if $a<\tau_{1}$, since $u\left(a^{+}\right)=u\left(\tau_{1}\right)=1, u^{\prime}(t) \leq 0$ in $\left(a, \tau_{1}\right)$, then necessarily $u^{\prime} \equiv 0, u \equiv 1$ in $\left(a, \tau_{1}\right)$ and $a=-\infty$, since $I=(a, b)$ the maximal existence interval of the solution. The case $\tau_{2}<b$ is analogous.

Step 2. If problem (E), (2.2)-(2.3) is solvable, then $\alpha<\beta$.

Let us assume $\alpha=\beta$. Since $u^{\prime}(t)<0$ in $\left(\tau_{1}, T_{1}\right) \cup\left(T_{2}, \tau_{2}\right)$, there exists the inverse functions $t_{1}=t_{1}(u):(\alpha, 1) \mapsto\left(\tau_{1}, T_{1}\right), t_{2}=t_{2}(u):(0, \alpha) \mapsto\left(T_{2}, \tau_{2}\right)$. Let $z_{1}(u):=D(u) u^{\prime}\left(t_{1}(u)\right), u \in(\alpha, 1)$ and $z_{2}(u):=D(u) u^{\prime}\left(t_{2}(u)\right), u \in(0, \alpha)$. Clearly $z_{1}(u)>0$ in $(\alpha, 1), z_{1}\left(\alpha^{+}\right)=0$ since $D(\alpha)=0, z_{1}\left(1^{-}\right)=0$ by $(2.3)$ if $\tau_{1}=a$ or by Step 1 if $\tau_{1}>a$. Similarly, $z_{2}(u)<0$ in $(0, \alpha), z_{2}\left(\alpha^{-}\right)=0$ since $D(\alpha)=0$, $z_{2}\left(0^{+}\right)=0$ by $(2.3)$ if $\tau_{2}=b$ or by Step 1 if $\tau_{2}<b$. Further, both $z_{1}$ and $z_{2}$ satisfy the equation

$$
\dot{z}(u)=-c-\frac{f(u) D(u)}{z(u)}
$$

in $(\alpha, 1)$ and $(0, \alpha)$ respectively. Multiplying by $z(u)$ and integrating the equality $z_{j} \dot{z}_{j}=-c z_{j}-f(u) D(u)$, in $(\alpha, 1)$ if $j=1$, and in $(0, \alpha)$ if $j=2$, we obtain

$$
c=\frac{-\int_{0}^{\alpha} f(u) D(u) d u}{\int_{0}^{\alpha} z_{2}(u) d u}=\frac{-\int_{\alpha}^{1} f(u) D(u) d u}{\int_{\alpha}^{1} z_{1}(u) d u} .
$$

Since $f(u) D(u)<0$ and $z_{1}(u)>0$ in $(\alpha, 1)$ while $f(u) D(u)<0$ and $z_{2}(u)<0$ in $(0, \alpha)$, we obtain a contradiction.

Assume now, again by contradiction, $\alpha>\beta$. By means of the same argument as previously, consider the inverse functions $t_{1}=t_{1}(u):(\alpha, 1) \mapsto\left(\tau_{1}, T_{1}\right), t_{2}=$ $t_{2}(u):(0, \beta) \mapsto\left(T_{2}, \tau_{2}\right)$, and the functions $z_{1}(u)=D(u) u^{\prime}\left(t_{1}(u)\right), u \in(\alpha, 1)$ and $z_{2}(u)=D(u) u^{\prime}\left(t_{2}(u)\right), u \in(0, \beta)$. Since $z_{2}$ satisfies $(2.5)$ in its existence interval, integrating the relation $z_{2}(u) \dot{z}_{2}(u)=-c z_{2}(u)-f(u) D(u)$ in $(0, \beta)$ we obtain

$$
c=\frac{-\int_{0}^{\beta} f(u) D(u) d u}{\int_{0}^{\beta} z_{2}(u) d u}<0
$$

taking into account that $f(u) D(u)<0, z_{2}(u)<0$ in $(0, \beta)$. Further, $z_{1}(u)>0$ in $(\alpha, 1)$ and from $(2.5), \dot{z}_{1}(u)>-c>0$ in $(\alpha, 1)$, in contradiction with the boundary value $z_{1}\left(1^{-}\right)=0$. 
Step 3. If problem (E), (2.2)-(2.3) is solvable, then $c>0$.

With the same argument as above consider the inverse function $t_{1}=t_{1}(u)$ : $(\beta, 1) \mapsto\left(\tau_{1}, T_{1}\right)$, and $z_{1}(u)=D(u) u^{\prime}\left(t_{1}(u)\right), u \in(\beta, 1)$. Since $z_{1}(u)>0$ in $(\beta, 1)$, it satisfies $(2.5)$ in this interval, and $z_{1}\left(\beta^{+}\right)=z_{1}\left(1^{-}\right)=0$, so integrating the relation $\dot{z}_{1}(u) z_{1}(u)=-c z_{1}(u)-f(u) D(u)$ on $(\beta, 1)$, we obtain

$$
c=\frac{-\int_{\beta}^{1} f(u) D(u) d u}{\int_{\beta}^{1} z_{1}(u) d u}>0 .
$$

Step 4. If $u$ is a solution of problem (E), (2.2)-(2.3), then $u^{\prime}(t)<0$ for every $t \in\left(\tau_{1}, \tau_{2}\right)$.

Let us consider the function $z_{2}(u)=D(u) u^{\prime}\left(t_{2}(u)\right), u \in(0, \alpha)$ defined in Step 2. We have $z_{2}(u)<0$ in $(0, \alpha)$ and $z_{2}\left(0^{+}\right)=0$. Moreover, by $(2.5)$ we have $\dot{z}_{2}(u)<-c<0$ in $(0, \alpha)$ and so $z_{2}\left(\alpha^{-}\right)<0$. Since $D(\alpha)>0$, this implies $u^{\prime}\left(T_{2}\right)<0$. Moreover, since $u\left(T_{1}\right)=\beta$ and $D(\beta)=0$, we immediately get $h^{\prime}\left(T_{1}\right)=\dot{D}(\beta)\left[u^{\prime}\left(T_{1}\right)\right]^{2}$, where $h$ was defined in Step 1, and from (E) it follows that $\dot{D}(\beta)\left[u^{\prime}\left(T_{1}\right)\right]^{2}+c u^{\prime}\left(T_{1}\right)+f(\beta)=0$. Hence, since $f(\beta) \neq 0$ and $u^{\prime}\left(T_{1}\right) \leq 0$, we get $u^{\prime}\left(T_{1}\right)<0$. Note that the same argument shows that $u^{\prime}(t) \neq 0$ whenever $u(t)=\beta$.

Assume now, by contradiction, that $J_{0}:=\left\{t \in\left(T_{1}, T_{2}\right): u^{\prime}(t)=0\right\} \neq \emptyset$. Let $s_{1}=\min J_{0}, s_{2}=\max J_{0}$. Notice that if $t_{0} \in J_{0}$ with $u\left(t_{0}\right) \in(0, \alpha) \cup(\beta, 1)$, then it is a proper local minimum for $u$, while if $u\left(t_{0}\right) \in(\alpha, \beta)$ then it is a proper local maximum for $u$. Further, $u^{\prime}(s) \neq 0$ for every $s$ such that $u(s)=\beta$. Therefore $u\left(s_{1}\right) \in[0, \alpha]$, while $u\left(s_{2}\right) \in(\alpha, \beta)$ or $u\left(s_{2}\right)=1$. So, in particular, $s_{1} \neq s_{2}$.

Let us now show that $u\left(s_{2}\right) \in(\alpha, \beta)$. To this aim, assume by contradiction that $u\left(s_{2}\right)=1$. Since in $(\beta, 1)$ there can only be minima for $u(t)$, there exists an interval $\left(s_{3}, \sigma_{3}\right) \subseteq\left(s_{1}, s_{2}\right)$ such that $u\left(s_{3}\right)=\beta, u\left(\sigma_{3}\right)=1, u^{\prime}(t)>0$ in $\left(s_{3}, \sigma_{3}\right)$. Let us consider the inverse function $t_{3}(u):(\beta, 1) \mapsto\left(s_{3}, \sigma_{3}\right)$ and let $\zeta_{3}(u):=D(u) u^{\prime}\left(t_{3}(u)\right)$ for $u \in(\beta, 1)$.

We have that $\zeta_{3}$ is a solution in $(\beta, 1)$ of Eq. $(2.5)$, with $\zeta_{3}(\beta)=0$ since $D(\beta)=0$, and $\zeta_{3}\left(1^{-}\right)=0$ since $u^{\prime}\left(\sigma_{3}\right)=0$. So, integrating the equation $\dot{\zeta}_{3} \zeta_{3}=-c \zeta_{3}-$ $f(u) D(u)$ in $(\beta, 1)$ we obtain

$$
c=\frac{-\int_{\beta}^{1} f(u) D(u) d u}{\int_{\beta}^{1} \zeta_{3}(u) d u} .
$$

Since $\zeta_{3}(u)$ and $f(u) D(u)$ are both negative in $(\beta, 1)$, this implies $c<0$ in contradiction to Step 3 ; therefore $u\left(s_{2}\right) \in(\alpha, \beta)$.

Since $u^{\prime}(t)<0$ in $\left(T_{1}, s_{1}\right)$ and in $\left(s_{2}, \tau_{2}\right)$, then there exist the inverse functions $t_{1}(u):\left(u\left(s_{1}\right), \beta\right) \mapsto\left(T_{1}, s_{1}\right)$ and $t_{2}(u):\left(0, u\left(s_{2}\right)\right) \mapsto\left(s_{2}, \tau_{2}\right)$. Let $z_{1}(u)=$ $D(u) u^{\prime}\left(t_{1}(u)\right)$ for $u \in\left(u\left(s_{1}\right), \beta\right)$, and $z_{2}(u)=D(u) u^{\prime}\left(t_{2}(u)\right)$, for $u \in\left(0, u\left(s_{2}\right)\right)$. Then both $z_{1}$ and $z_{2}$ are negative and satisfy (2.5) in their existence interval. Since $u^{\prime}\left(s_{1}\right)=u^{\prime}\left(s_{2}\right)=0$, then $z_{1}\left(u\left(s_{1}\right)^{+}\right)=0=z_{2}\left(u\left(s_{2}\right)^{-}\right)$; further $z_{1}\left(\beta^{-}\right)=0$ since $D(\beta)=0$, and $z_{2}\left(0^{+}\right)=0$ since if $\tau_{2}<b$ we have $u^{\prime}\left(\tau_{2}\right)=0$, while if $\tau_{2}=b$ it follows from (2.3). 
If $u\left(s_{1}\right)>0$, a point $\bar{u} \in\left(u\left(s_{1}\right), u\left(s_{2}\right)\right) \subset(0, \beta)$ should exist such that $z_{1}(\bar{u})=$ $z_{2}(\bar{u})<0$, but this is impossible, since $(2.5)$ has the uniqueness property for Cauchy problems with initial data different from zero.

If $u\left(s_{1}\right)=0$, then $z_{1}$ and $z_{2}$ should be two negative solutions of Eq. (2.5) in their existence intervals, $(0, \beta)$ and $\left(0, u\left(s_{2}\right)\right)$ respectively, with $0=z_{2}\left(u\left(s_{2}\right)^{-}\right)>$ $z_{1}\left(u\left(s_{2}\right)\right)$. So, again by the above-mentioned uniqueness property for Eq. (2.5), we deduce that $z_{1}(u)<z_{2}(u)$ for every $u \in\left(0, u\left(s_{2}\right)\right)$.

Put $\zeta(u):=z_{1}(u)-z_{2}(u), u \in(0, \alpha]$, we have $\zeta\left(0^{+}\right)=0$ (since $z_{1}\left(0^{+}\right)=$ $\left.z_{2}\left(0^{+}\right)=0\right), \zeta(u)<0$ in $(0, \alpha]$ and $\dot{\zeta}(u)=\zeta(u) f(u) D(u) /\left(z_{1}(u) z_{2}(u)\right)$.

Let $F(u):=\exp \left(\int_{u}^{\alpha} \frac{f(v) D(v)}{z_{1}(v) z_{2}(v)} d v\right), u \in(0, \alpha]$. Since $f(u) D(u)<0$ in $(0, \alpha)$, there exists $F\left(0^{+}\right) \in[0,1)$. Moreover $\frac{d}{d u} \zeta(u) F(u)=0$, hence $\zeta(u) F(u) \equiv 0$ in $(0, \alpha]$. As $F(u)>0$, this implies $\zeta(u) \equiv 0$ in $(0, \alpha]$, a contradiction, and this concludes the proof of Step 4.

Step 5. If problem (E), (2.2)-(2.3) is solvable, then

$$
\int_{0}^{\beta} f(u) D(u) d u>0 .
$$

Since $u^{\prime}(t)<0$ in $\left(\tau_{1}, \tau_{2}\right)$, we can consider the inverse function $t(u):(0,1) \mapsto\left(\tau_{1}, \tau_{2}\right)$ and $z(u)=D(u) u^{\prime}(t(u))$. Of course, $z \in C^{1}(0,1), z(u)<0$ in $(0, \beta)$ and $z$ satisfies $(2.5)$ in this interval. Further, $z\left(0^{+}\right)=D(0) u^{\prime}\left(\tau_{2}\right)=0$ if $\tau_{2}<b$, while if $\tau_{2}=b$ then $z\left(0^{+}\right)=0$ by $(2.3)$. Since $c>0$ by Step 3 , and

$$
c=\frac{-\int_{0}^{\beta} f(u) D(u) d u}{\int_{0}^{\beta} z(u) d u}
$$

we immediately obtain

$$
\int_{0}^{\beta} f(u) D(u) d u>0 .
$$

\section{Existence and Non-Existence of t.w.s.}

Throughout this section assume that there exist (finite or infinite) the limits

$$
\lim _{u \rightarrow 0^{+}} \frac{f(u) D(u)}{u} \geq-\infty, \quad \lim _{u \rightarrow 1^{-}} \frac{f(u) D(u)}{u-1} \leq+\infty .
$$

Put $g(u):=f(u) D(u)$, and let us now consider the equation

$$
u^{\prime \prime}+c u^{\prime}+g(u)=0, \quad u \in(0, \beta) .
$$

Note that $g(u)$ is a Nagumo-type reaction term in $(0, \beta)$. Therefore, ${ }^{6}$ if $\int_{0}^{\beta} g(u) d u>$ 0 , there exists a unique positive value $c_{1}^{*}$, satisfying the estimate

$$
c_{1}^{*}<2 \sqrt{\sup _{u \in(\alpha, \beta]} \frac{g(u)}{u-\alpha}}
$$

such that (3.2) admits $C^{2}$-solutions in $\mathbb{R}$, satisfying the boundary conditions $u(-\infty)=\beta, u(+\infty)=0$. Moreover, the solution is unique, up to translation. 
Similarly, put $\tilde{g}(u):=-g(1-u)$, and consider the equation

$$
u^{\prime \prime}+c u^{\prime}+\tilde{g}(u)=0, \quad u \in(0,1-\beta) .
$$

In this case the function $\tilde{g}(u)$ is a Fisher-type reaction term and, when $\tilde{g}^{\prime}(0)<+\infty$ (i.e. $\left.g^{\prime}(1)<+\infty\right)$, there exists a positive value $c_{2}^{*},{ }^{6}$ satisfying the estimate

$$
2 \sqrt{\tilde{g}^{\prime}(0)} \leq c_{2}^{*} \leq 2 \sqrt{\sup _{s \in(0,1-\beta]} \frac{\tilde{g}(s)}{s}},
$$

i.e.

$$
2 \sqrt{g^{\prime}(1)} \leq c_{2}^{*} \leq 2 \sqrt{\sup _{u \in[\beta, 1)} \frac{g(u)}{u-1}},
$$

such that (3.4) admits $C^{2}$-solutions in $\mathbb{R}$ satisfying the boundary conditions $u(-\infty)=1-\beta, u(+\infty)=0$, if and only if $c \geq c_{2}^{*}$ (note that $g^{\prime}(1)$ exists, finite or infinite, by (3.1)). Moreover, for any admissible $c$, the solution is unique up to translation.

The solvability of problem (E), (2.2)-(2.3) is linked to the values of $c_{1}^{*}$ and $c_{2}^{*}$, as the following result shows.

Theorem 3.1. Equation (E) admits solutions satisfying conditions (2.2)-(2.3), if and only if

$$
\alpha<\beta, \quad c>0, \quad \int_{0}^{\beta} f(u) D(u) d u>0
$$

and

$$
c_{1}^{*} \geq c_{2}^{*}
$$

In this case, there exist solutions only for $c=c_{1}^{*}$ and the solution is unique, up to translation. Moreover, the solution is

- sharp of type (I) if and only if $D(0)=0$ and $c_{1}^{*}>c_{2}^{*}$ or $c_{1}^{*}=c_{2}^{*}$ but $D(1)<0$;

- sharp of type (II) if and only if $D(0)>0, D(1)=0$ and $c_{1}^{*}=c_{2}^{*}$;

- sharp of type (III) if and only if $D(0)=D(1)=0$ and $c_{1}^{*}=c_{2}^{*}$;

- front-type in the remaining cases.

Proof. Again we divide the proof into several steps.

Step 1. There is a bijection between the set of solutions of problem (E), (2.2)-(2.3), modulo translation of the origin, and the functions $z \in C^{1}(0,1)$, satisfying $(2.5)$ in $(0, \beta) \cup(\beta, 1)$, and such that $z(u)<0$ in $(0, \beta), z(u)>0$ in $(\beta, 1), z\left(0^{+}\right)=z\left(1^{-}\right)=0$.

In Step 5 of Theorem 2.1, we proved that if $u$ is a solution of problem (E), (2.2)(2.3), then the function $z(u)=D(u) u^{\prime}(t(u)$ ) (where $t(u)$ is the inverse function of $\left.u(t), t \in\left(\tau_{1}, \tau_{2}\right)\right)$ is in $C^{1}(0,1)$, and satisfies $(2.5)$ in $(0, \beta)$. Moreover $z(u)<0$ in this interval, and $z\left(0^{+}\right)=0$. One can immediately check that $z(u)$ satisfies $(2.5)$ also in $(\beta, 1), z(u)>0$ in this interval, and $z\left(1^{-}\right)=0$. 
Vice versa, let $z \in C^{1}(0,1)$, satisfying $(2.5)$ in $(0, \beta) \cup(\beta, 1)$, and such that $z(u)<0$ in $(0, \beta), z(u)>0$ in $(\beta, 1), z\left(0^{+}\right)=z\left(1^{-}\right)=0$. First note that if $\dot{D}(\beta) \neq 0$ then $\dot{z}(\beta)>0$, while $\dot{D}(\beta)=0$ implies $\dot{z}(\beta)=0$. Indeed, from (2.5), we have

$$
\dot{z}(\beta)[\dot{z}(\beta)+c]=\lim _{u \rightarrow \beta} \frac{z(u)(\dot{z}(u)+c)}{u-\beta}=\lim _{u \rightarrow \beta} \frac{-g(u)}{u-\beta}=-f(\beta) \dot{D}(\beta) .
$$

Therefore, since $\dot{z}(\beta) \geq 0$ and $c>0$, we get $\dot{z}(\beta)=0$ if and only if $\dot{D}(\beta)=0$. Further, let us prove that the $\operatorname{limit}_{\lim _{u \rightarrow \beta}} z(u) / D(u)=: \ell$ exists and is finite. This is immediate when $\dot{D}(\beta) \neq 0$, so consider the case $\dot{D}(\beta)=0$. Given $\epsilon>0$, since $c>0$, it is possible to find $0<\delta_{1}<\beta-\alpha$ satisfying

$$
-c+\frac{c f(u)}{f(\beta)+c \epsilon}+\left(\frac{f(\beta)}{c}+\epsilon\right) \dot{D}(u)<0, \quad \beta-\delta_{1} \leq u \leq \beta .
$$

We claim that

$$
z(u)>\tilde{z}(u):=-\left(\frac{f(\beta)}{c}+\epsilon\right) D(u), \quad \beta-\delta_{1}<u<\beta .
$$

In fact, assuming $\tilde{z}\left(u_{0}\right) \geq z\left(u_{0}\right)$ for some $u_{0} \in\left(\beta-\delta_{1}, \beta\right)$, we obtain by $(3.7)$

$$
\dot{z}\left(u_{0}\right)=-c-\frac{f\left(u_{0}\right) D\left(u_{0}\right)}{z\left(u_{0}\right)} \leq-c+\frac{c f\left(u_{0}\right)}{f(\beta)+c \epsilon}<-\left(\frac{f(\beta)}{c}+\epsilon\right) \dot{D}\left(u_{0}\right)=\dot{\tilde{z}}\left(u_{0}\right),
$$

implying the contradictory conclusion $z\left(\beta^{-}\right)<\tilde{z}\left(\beta^{-}\right)=0$. Hence (3.8) is valid. With a similar reasoning we are able to find $0<\delta_{2}<\beta-\alpha$ such that

$$
z(u)<-\left(\frac{f(\beta)}{c}-\epsilon\right) D(u), \quad \beta-\delta_{2}<u<\beta,
$$

for $\epsilon$ sufficiently small. Let $\delta:=\min \left\{\delta_{1}, \delta_{2}\right\}$. According to (3.8) and (3.9) we have

$$
-\frac{f(\beta)}{c}-\epsilon \leq \frac{z(u)}{D(u)} \leq-\frac{f(\beta)}{c}+\epsilon, \quad \beta-\delta<u<\beta .
$$

Since $\epsilon$ is an arbitrary positive value, we get $\lim _{u \rightarrow \beta^{-}} z(u) / D(u)=-f(\beta) / c$. Similarly, one can also prove that $\lim _{u \rightarrow \beta^{+}} z(u) / D(u)=-f(\beta) / c$ when $\dot{D}(\beta)=0$.

Now, let $u$ be the unique solution of the Cauchy problem

$$
\left\{\begin{array}{ll}
u^{\prime}=G(u) \\
u(0)=1 / 2
\end{array} \quad \text { where } G(u)= \begin{cases}z(u) / D(u), & u \in(0,1), \quad u \neq \beta \\
\ell, & u=\beta\end{cases}\right.
$$

defined in its maximal existence interval $\left(\tau_{1}, \tau_{2}\right)$. Notice that $G \in C^{0}(0,1)$ and $D(u(t)) u^{\prime}(t)=z(u(t))$ for every $t \in\left(\tau_{1}, \tau_{2}\right)$, so $D(u) u^{\prime} \in C^{1}\left(\tau_{1}, \tau_{2}\right)$ with $u^{\prime}(t)<0$ and $\lim _{t \rightarrow \tau_{1}^{+}} D(u(t)) u^{\prime}(t)=\lim _{u \rightarrow 1^{-}} z(u)=0, \lim _{t \rightarrow \tau_{2}^{-}} D(u(t)) u^{\prime}(t)$ $=\lim _{u \rightarrow 0^{+}} z(u)=0$. Further,

$$
\left(D(u) u^{\prime}\right)^{\prime}(t)=\dot{z}(u(t)) u^{\prime}(t)=-c u^{\prime}(t)-f(u(t)) .
$$


$u$ is a solution of $(\mathrm{E}),(2.2)-(2.3)$ in $\left(\tau_{1}, \tau_{2}\right)$. If we denote by $I=(a, b)$ the maximal existence interval of $u(t)$ as a solution of $(\mathrm{E}),(2.2)-(2.3)$, then the values $\tau_{1}, \tau_{2}$ have the same meaning as at the beginning of the proof of Theorem 2.1.

Step 2. Equation (E) admits solutions satisfying conditions (2.2)-(2.3), if and only if $c_{1}^{*} \geq c_{2}^{*}$.

By virtue of Step 1, the solvability of problem (E), (2.2)-(2.3) is equivalent to the existence of functions $z \in C^{1}(0,1)$, satisfying $(2.5)$ in $(0, \beta) \cup(\beta, 1)$, such that $z\left(0^{+}\right)=z\left(1^{-}\right)=0$ and $z(u)<0$ in $(0, \beta), z(u)>0$ in $(\beta, 1)$. Observe now that the existence of negative solutions of $(2.5)$ in $(0, \beta)$, satisfying $z\left(0^{+}\right)=z\left(\beta^{-}\right)=0$ is equivalent to the existence of solutions for (3.2) satisfying $u(-\infty)=\beta, u(+\infty)=0 .{ }^{11}$ Similarly, it is easy to check that the existence of positive solutions of $(2.5)$ in $(\beta, 1)$, satisfying $z\left(\beta^{+}\right)=z\left(1^{-}\right)=0$ is equivalent to the existence of negative solutions of the equation

$$
\dot{w}=-c-\frac{\tilde{g}(s)}{w(s)}, \quad s \in(0,1-\beta)
$$

satisfying $w\left(0^{+}\right)=w\left((1-\beta)^{-}\right)=0$; indeed it suffices to put $w(s)=-z(1-s)$ (recall that $\tilde{g}(s)=-g(1-s)$ ). Further, this is equivalent to the existence of $C^{2}$ solutions $u(t)$ of $(3.4)$, satisfying $u(-\infty)=1-\beta, u(+\infty)=0$. Therefore, if problem (E), (2.2)-(2.3) is solvable for some $c$, then $c=c_{1}^{*}$, and $c_{1}^{*} \geq c_{2}^{*}$.

Vice versa, if $\alpha<\beta$ and $\int_{0}^{\beta} f(u) D(u) d u>0$, then $c_{1}^{*}$ and $c_{2}^{*}$ exist and assuming $c_{1}^{*} \geq c_{2}^{*}$, then for $c=c_{1}^{*}$ there exists a positive function $z_{2} \in C^{1}(\beta, 1)$, solution of $(2.5)$ in $(\beta, 1)$, satisfying $z_{2}\left(\beta^{+}\right)=z_{2}\left(1^{-}\right)=0$ and there exists a negative function $z_{1} \in C^{1}(0, \beta)$, solution of $(2.5)$ in $(0, \beta)$, satisfying $z_{1}\left(0^{+}\right)=z_{1}\left(\beta^{-}\right)=0$. In order to conclude the proof, it is sufficient to show that the function $z(u)$, defined by

$$
z(u)= \begin{cases}z_{1}(u), & u \in(0, \beta) \\ 0, & u=\beta \\ z_{2}(u), & u \in(\beta, 1)\end{cases}
$$

satisfies $z \in C^{1}(0,1)$. To this aim, assume by contradiction

$$
\limsup _{u \rightarrow \beta^{+}} \frac{z(u)}{u-\beta}:=D^{+} z(\beta)>D_{+} z(\beta):=\limsup _{u \rightarrow \beta^{-}} \frac{z(u)}{u-\beta}
$$

and fix $\lambda \in\left(D_{+} z(\beta), D^{+} z(\beta)\right)$. Let $\left(u_{n}\right)_{n}$ be a decreasing sequence converging to $\beta$, such that

$$
\frac{z\left(u_{n}\right)}{u_{n}-\beta}=\lambda, \quad \text { and }\left.\quad \frac{d}{d u}\left(\frac{z(u)}{u-\beta}\right)\right|_{u=u_{n}} \leq 0 .
$$

Since $\frac{d}{d u} \frac{z(u)}{u-\beta}=\frac{1}{u-\beta}\left(\dot{z}(u)-\frac{z(u)}{u-\beta}\right)$, we have $\dot{z}\left(u_{n}\right)=-c-\frac{g\left(u_{n}\right)}{\lambda\left(u_{n}-\beta\right)} \leq \lambda$. Passing to the limit as $n \rightarrow+\infty$, since $\lambda>0$, we get $\lambda^{2}+c \lambda+\dot{g}(\beta) \geq 0$. Similarly, if we choose a decreasing sequence $\left(v_{n}\right)_{n}$ converging to $\beta$, such that

$$
\frac{z\left(v_{n}\right)}{v_{n}-\beta}=\lambda, \quad \text { and }\left.\quad \frac{d}{d u}\left(\frac{z(u)}{u-\beta}\right)\right|_{u=v_{n}} \geq 0,
$$


we can deduce that $\lambda^{2}+c \lambda+\dot{g}(\beta) \leq 0$. Therefore, we obtain $\lambda^{2}+c \lambda+\dot{g}(\beta)=0$ and a contradiction follows from the arbitrariness of $\lambda \in\left(D_{+} z(\beta), D^{+} z(\beta)\right)$. This proves the existence of the $\operatorname{limit}_{\lim _{u \rightarrow \beta^{+}}} z(u) /(u-\beta) \in[0,+\infty]$. If $\dot{D}(\beta) \neq 0$, from (2.5) we deduce

$$
\dot{z}\left(\beta^{+}\right)=-c-f(\beta) \dot{D}(\beta) \lim _{u \rightarrow \beta^{+}} \frac{u-\beta}{z(u)} .
$$

Notice that, if $\lim _{u \rightarrow \beta^{+}} z(u) /(u-\beta)=0(+\infty)$ then $\dot{z}\left(\beta^{+}\right)=+\infty(0)$ and this is not possible. Hence $\dot{z}\left(\beta^{+}\right)$exists and it has the real value $\frac{1}{2}\left(-c+\sqrt{c^{2}-4 f(\beta) \dot{D}(\beta)}\right)$. On the other hand, if $\dot{D}(\beta)=0$, by what we proved in Step 1 we get $\lim _{u \rightarrow \beta^{+}} \frac{D(u)}{z(u)}=$ $-\frac{c}{f(\beta)}$. Hence, from (2.5) we deduce that $\dot{z}\left(\beta^{+}\right)=-c-f(\beta) \lim _{u \rightarrow \beta^{+}} \frac{D(u)}{z(u)}=0$. Similarly one can prove the existence of the limit $\dot{z}\left(\beta^{-}\right)$, coincident with $\dot{z}\left(\beta^{+}\right)$. Consequently Eq. (E) with $c=c_{1}^{*}$ admits a solution satisfying (2.2)-(2.3).

Finally, the uniqueness of the solution (up to translation) derives from the uniqueness of the solutions of Eqs. (3.2), (3.4) satisfying the prescribed boundary conditions.

Step 3. Characterization of the t.w.s.

Let $u(t)$ be the solution of problem (E), (2.2)-(2.3) with $c=c_{1}^{*}$ as shown in Step 2. In order to distinguish what kind of solution $u(t)$ is, we need to calculate

$$
u^{\prime}\left(a^{+}\right)=\lim _{t \rightarrow a^{+}} u^{\prime}(t)=\lim _{u \rightarrow 1^{-}} \frac{z(u)}{D(u)}
$$

and

$$
u^{\prime}\left(b^{-}\right)=\lim _{t \rightarrow b^{-}} u^{\prime}(t)=\lim _{u \rightarrow 0^{+}} \frac{z(u)}{D(u)},
$$

where $z(u)$ is the associated first-order dynamics (see Step 1) satisfying $z\left(0^{+}\right)=$ $z\left(1^{-}\right)=0$. When $D(1) \neq 0$, it follows that $u^{\prime}\left(a^{+}\right)=0$ hence $a=-\infty$ and the solution is classical for $u$ near its value 1 . Similarly, $D(0) \neq 0$ implies $u^{\prime}\left(b^{-}\right)=0$ hence $b=+\infty$ and again the solution is classical near $u=0$. Consequently, whenever $D(0) D(1) \neq 0$, both $a=-\infty$ and $b=+\infty$ are valid, hence $u(t)$ is a front-type solution.

Consider now the case when $D(1)=0$. Since $c_{1}^{*} \geq c_{2}^{*}$, the following problem is uniquely solvable

$$
\left\{\begin{array}{l}
\dot{w}(s)=-c_{1}^{*}+\frac{f(1-s) D(1-s)}{w(s)}, \quad s \in(0,1-\beta) \\
w(s)<0 \\
w\left(0^{+}\right)=w\left((1-\beta)^{-}\right)=0
\end{array}\right.
$$

with $w(s)$ defined as in Step 2. Reasoning as in Ref. 10, Lemma 5 (see also Ref. 9, Theorem 4) it is possible to prove that the limit $\dot{w}(0)=\lim _{s \rightarrow 0^{+}} w(s) / s$ exists and it is 0 or $-c_{1}^{*}$. In particular, $\dot{w}(0)=0$ if and only if $c_{1}^{*}>c_{2}^{*}$ (see Ref. 10, Corollary 11). 
First assume $c_{1}^{*}>c_{2}^{*}$ and show that, in this case, $u^{\prime}\left(a^{+}\right)=0$. Indeed, this is trivial when $\dot{D}(1) \neq 0$ since $z(u)=-w(1-u)$ implies $\dot{z}(1)=0$ and

$$
u^{\prime}\left(a^{+}\right)=\lim _{u \rightarrow 1^{-}} \frac{z(u)}{u} \frac{u}{D(u)} .
$$

Instead, when $\dot{D}(1)=0$ according to the proof of Theorem 4 in Ref. 9, we have

$$
u^{\prime}\left(a^{+}\right)=\lim _{u \rightarrow 1^{-}} \frac{z(u)}{D(u)}=\lim _{s \rightarrow 0^{+}} \frac{w(s)}{D(1-s)}=0 .
$$

Consequently $a=-\infty$ and again when $D(0) \neq 0$ the solution $u(t)$ is of front-type. Now consider $c_{1}^{*}=c_{2}^{*}$. Since $\dot{z}(1)=\dot{w}(0)=-c_{1}^{*}$, we obtain

$$
\lim _{t \rightarrow a^{+}} u^{\prime}(t)=\lim _{u \rightarrow 1^{-}} \frac{z(u)}{D(u)}=\lim _{u \rightarrow 1^{-}} \frac{z(u)}{u-1} \frac{u-1}{D(u)}=-\frac{c_{1} *}{\dot{D}(1)},
$$

with the obvious meaning that it is $-\infty$ when $\dot{D}(1)=0$. Therefore $a>-\infty$ and when $D(0)>0, b=+\infty$ hence $u(t)$ is a sharp solution of type (II).

It remains to consider the case when $D(0)=0$. Since $z(u)$ satisfies $(2.5)$ in $(0, \alpha)$ with $c=c_{1}^{*}$, we obtain

$$
\lim _{u \rightarrow 0^{+}}\left[\dot{z}(u)+c_{1}^{*}\right] \frac{z(u)}{u}=\lim _{u \rightarrow 0^{+}}-f(u) \frac{D(u)}{u}=0 .
$$

We recall that $z(u)$ and $f(u) D(u)$ are negative in $(0, \alpha)$, so by $(2.5)$ it follows that $\dot{z}(u)<-c_{1}^{*}$ in $(0, \alpha)$; this implies $\lim _{u \rightarrow 0^{+}} \dot{z}(u)=-c_{1}^{*}$, since at $u=0$ we have $\dot{z}(0)=0$ or $\dot{z}(0)=-c_{1}^{*}$. Therefore

$$
\lim _{u \rightarrow b^{-}} u^{\prime}(t)=\lim _{u \rightarrow 0^{+}} \frac{z(u)}{D(u)}=\lim _{u \rightarrow 0^{+}} \frac{\dot{z}(u)}{\dot{D}(u)}=-\frac{c_{1}^{*}}{\dot{D}(0)}
$$

with the obvious meaning that the limit is $-\infty$ when $\dot{D}(1)=0$; in every case $b<+\infty$. Consequently, when $c_{1}^{*}>c_{2}^{*}$ or $c_{1}^{*}=c_{2}^{*}$ but $D(1)<0$, then $a=-\infty$ and $u(t)$ is sharp of type (I) while when $c_{1}^{*}=c_{2}^{*}$ and $D(1)=0$ also $a>-\infty$ implying that $u(t)$ is sharp of type (III).

As is natural, the necessary and sufficient condition stated in the previous result has an implicit form, since the values of the constants $c_{1}^{*}$ and $c_{2}^{*}$ are unknown in general. So, in order to make it applicable, we now give some simple conditions, directly expressed in terms of the coefficients $D(u)$ and $f(u)$, ensuring the validity of condition (3.6).

Theorem 3.2. Assume

$$
\lim _{u \rightarrow 1^{-}} \frac{f(u) D(u)}{u-1} \geq \sup _{u \in(\alpha, \beta]} \frac{f(u) D(u)}{u-\alpha} .
$$

Then Eq. (E) does not admit solutions satisfying (2.2)-(2.3), for any value $c>0$. Instead, if all the necessary conditions (2.4) hold, put

$$
\sigma:=\sup _{u \in[\beta, 1)} \frac{f(u) D(u)}{u-1}, \quad m:=-\min _{u \in[0, \alpha]} f(u) D(u)
$$


then, if

$$
8 \sigma \beta^{2}+4 \sqrt{4 \beta^{4} \sigma^{2}+2 m \sigma \beta^{3}} \leq 3 \int_{0}^{\beta} f(u) D(u) d u
$$

there exists a unique value $c^{*}$, satisfying estimate (3.3), such that Eq. (E) admits a unique (up to translation) solution satisfying (2.2)-(2.3), which is sharp of type (I) if $D(0)=0$, and of front-type if $D(0)>0$.

Proof. From the estimates $(3.3),(3.5)$ of the critical values $c_{1}^{*}, c_{2}^{*}$, it follows immediately that a necessary condition for (3.6) to be satisfied is that

$$
\lim _{u \rightarrow 1^{-}} \frac{f(u) D(u)}{u-1}<\sup _{u \in(\alpha, \beta]} \frac{f(u) D(u)}{u-\alpha} .
$$

Assume now that all the conditions in (2.4) are satisfied and (3.13) holds. In Ref. 11, Lemma 4 , it was proved that for every $c>0$ there exists a unique negative solution $z_{c}$ of Eq. (2.5) in its maximal existence interval $\left(0, u_{c}\right)$, such that $z_{c}\left(0^{+}\right)=0$ and $\alpha<u_{c} \leq \beta$. Moreover, if $c_{1}<c_{2}$ then $u_{c_{1}} \leq u_{c_{2}}$ and $z_{c_{1}}(u)>z_{c_{2}}(u)$ in $\left(0, u_{c_{1}}\right)$.

For every $c>0$ let $k_{c}:=c \sqrt{\beta}+\sqrt{c^{2} \beta+2 m}$ and $\phi_{c}(u):=-k_{c} \sqrt{u}, u \in(0, \beta)$. Since $k_{c}^{2}=2 c k_{c} \sqrt{\beta}+2 m>2 c k_{c} \sqrt{u}+2 m$, we have

$$
\dot{\phi}_{c}(u)=-\frac{k_{c}}{2 \sqrt{u}}<-c-\frac{m}{k_{c} \sqrt{u}} \leq-c+\frac{g(u)}{k_{c} \sqrt{u}}=-c-\frac{g(u)}{\phi_{c}(u)},
$$

i.e. $\phi_{c}$ is a lower-solution for Eq. $(2.5)$ in $(0, \beta)$. Hence, by results proved in Ref. 11, Lemma 3 , we get $\phi_{c}(u) \leq z_{c}(u)$ in $\left(0, u_{c}\right)$. Moreover, since $\phi_{c}$ is a strict lowersolution, we have $\phi_{c}(u)<z_{c}(u)$ in $\left(0, u_{c}\right)$. Indeed, if $\phi_{c}(\bar{u})=z_{c}(\bar{u})$ for some $\bar{u} \in$ $\left(0, u_{c}\right)$, then $\dot{\phi}_{c}(\bar{u})<-c-\frac{g(\bar{u})}{\phi_{c}(\bar{u})}=-c-\frac{g(\bar{u})}{z_{c}(\bar{u})}=\dot{z}_{c}(\bar{u})$, implying $\phi_{c}(u)>z_{c}(u)$ in a left neighborhood of $\bar{u}$, a contradiction.

Hence, integrating equation $\dot{z}_{c}(u) z_{c}(u)=-c z_{c}(u)-g(u)$ in $\left(0, u_{c}\right)$ we obtain

$$
\begin{aligned}
0 \leq z_{c}^{2}\left(u_{c}^{-}\right) & =-2 c \int_{0}^{u_{c}} z_{c}(u) d u-2 \int_{0}^{u_{c}} g(u) d u \\
& <2 c k_{c} \int_{0}^{\beta} \sqrt{u} d u-2 \int_{0}^{u_{c}} g(u) d u \\
& =\frac{4}{3} c k_{c} \beta^{3 / 2}-2 \int_{0}^{u_{c}} g(u) d u,
\end{aligned}
$$

i.e.

$$
\int_{0}^{u_{c}} g(u) d u<\frac{2}{3} c k_{c} \beta^{3 / 2} \text { for every } c>0
$$

Therefore, if

$$
\frac{2}{3} c k_{c} \beta^{3 / 2} \leq \int_{0}^{\beta} g(u) d u,
$$

necessarily we have $u_{c}<\beta$ implying $c<c_{1}^{*}$. Note that for $c=2 \sqrt{\sigma}$ inequality (3.14) then reduces to (3.13), which then implies that $2 \sqrt{\sigma}<c_{1}^{*}$. Therefore, by (3.5) we obtain $c_{2}^{*}<c_{1}^{*}$ and by virtue of Theorem 3.1 we get the assertion. 
Example 1. Let us consider the equation

$$
\left((\beta-u) u^{\prime}\right)^{\prime}+c u^{\prime}+f(u)=0
$$

where $0<\beta<1$ and

$$
f(u)= \begin{cases}u(\beta+u)\left(u^{2}-\alpha^{2}\right), & u \in[0, \beta] \\ f(\beta) & u \in\left[\beta, u_{0}\right] \\ \frac{a^{2}}{4} \frac{1-u}{u-\beta}, & u \in\left(u_{0}, 1\right]\end{cases}
$$

with $0<\alpha<\beta / \sqrt{3}, a>0$ and $u_{0}=u_{0}(a) \in(\beta, 1)$ is the solution of the linear equation

$$
\frac{a^{2}}{4}(1-u)=f(\beta)(u-\beta)
$$

Of course, $f(u)$ is well-defined and continuous on all $[0,1]$; moreover it satisfies $f(u)(u-\alpha)>0$ in $(0,1) \backslash\{\alpha\}$. Consequently, (3.15) is a diffusion-aggregation process with a Nagumo-type reaction term, depending on the parameter $a$.

Equation (3.15) has a unique connection from its stationary states 0 and $\beta .^{6}$ Its speed $c_{1}^{*}$ is the same as the speed of the unique wave connection, in $[0, \beta]$, of

$$
u_{t}=u_{x x}+u\left(\beta^{2}-u^{2}\right)\left(u^{2}-\alpha^{2}\right) .
$$

Notice in fact that the wave profile can be obtained, in both cases, from the solutions of the following singular boundary value problem

$$
\left\{\begin{array}{l}
\dot{z}(u)=-c-\frac{u\left(\beta^{2}-u^{2}\right)\left(u^{2}-\alpha^{2}\right)}{z(u)}, \\
z\left(0^{+}\right)=z\left(\beta^{-}\right)=0 .
\end{array}\right.
$$

Consequently, by Ref. 6, Example 15, we get

$$
c_{1}^{*}=\frac{\beta^{2}-3 \alpha^{2}}{\sqrt{3}} .
$$

The constraint on $\alpha$ assures that $c_{1}^{*}>0$. It is also easy to see that $\int_{0}^{\beta} f(u)(\beta-$ u) $d u>0$.

In the interval $[\beta, 1]$, Eq. (3.15) supports infinitely many waves with a minimum speed $c_{2}^{*}$ satisfying $(3.5)$ where $g(u)$ is defined in $[\beta, 1]$ as follows

$$
g(u)= \begin{cases}f(\beta)(\beta-u), & u \in\left[\beta, u_{0}\right], \\ \frac{a^{2}}{4}(u-1), & u \in\left(u_{0}, 1\right] .\end{cases}
$$

Since $g$ is convex in $[\beta, 1]$ we get $c_{2}^{*}=2 \sqrt{g^{\prime}(1)}=a$. According to Theorem 3 , all the cases occur, concerning the existence of a wave connection, on varying of the parameter $a$. In particular, when

$$
\frac{\beta^{2}-3 \alpha^{2}}{\sqrt{3}} \geq a
$$


(3.15) has a unique wave connection from 0 to 1 with speed $c_{1}^{*}$. Its profile is of front-type, since $D(u)$ does not vanish in $u=0$ and $u=1$. Instead, in the opposite case when

$$
\frac{\beta^{2}-3 \alpha^{2}}{\sqrt{3}}<a,
$$

(3.15) does not support a wave connection from 0 to 1.

\section{Final Discussion}

In this paper, we have used upper and lower solutions and comparison techniques in the phase plane to investigate front propagation in a reaction-diffusion equation where the diffusion coefficient changes sign, while the reaction term is bi-stable. While the latter is a well-known model for the Allee effect, and the former can describe aggregation-diffusion, our goal is not to apply our results to a specific biological/ecological example, rather it is to extend the mathematical results available for such equations. Travelling wave phenomena arise in models for many areas of application, including population dynamics, morphogenesis, wound healing and cancer growth. In some of these cases, the models can be caricaturised such that they do have a complicated functional form of diffusion coefficient which changes sign. In such cases, our model may well capture the essence of the phenomena and our results may transfer across.

Due to the sign change of the diffusion term $D$ at the point $\beta$, the model supports a system of wave connections from 1 to $\beta$ and from $\beta$ to 0 . However, only when $\alpha<\beta$ ( $\alpha$ being the value at which the reaction term changes its sign) do there exist connections with the same positive wave speed. In this case, it is possible to "glue" them together, resulting in a wave between 1 and 0 . As a consequence of this analysis, a population can eventually propagate throughout its habitat (i.e. a front between 1 and 0 exists) only when aggregating movements prevail over random dispersion in a range of values where the population is increasing.

As far as we know, the description of the behavior of this system when random dispersion and the Allee effect are active together, is still an open question.

\section{References}

1. W. Alt, Models for mutual attraction and aggregation of motile individuals, in Mathematics in Biology and Medicine, Lecture Notes in Biomathematics, Vol. 57 (SpringerVerlag, 1985), pp. 33-38.

2. D. G. Aronson, The role of diffusion in mathematical population biology: Skellam revisited, in Mathematics in Biology and Medicine, Lecture Notes in Biomathematics, Vol. 57 (Springer-Verlag, 1985), pp. 2-6.

3. V. Capasso, D. Morale and K. Oelschläger, An interacting particle system modelling aggregation behavior: From individuals to populations, J. Math. Biol. 50 (2005) 49-66.

4. P. C. Fife and J. B. McLeod, A phase plane discussion of convergence to travelling fronts for nonlinear diffusion, Arch. Rational Mech. Anal. 75 (1980/81) 281-314. 
5. B. H. Gilding and R. Kersner, The characterization of reaction-convection-diffusion processes by travelling waves, J. Diff. Eqns. 124 (1996) 27-79.

6. B. H. Gilding and R. Kersner, Travelling Waves in Nonlinear Diffusion-ConvectionReaction, (Birkhäuser 2004).

7. P. Grindrod, Model of individual aggregation or clustering in single and multi-species communities, J. Math. Biol. 26 (1988) 651-660.

8. S. Kamin and P. Rosenau, Emergence of waves in a nonlinear convection-reactiondiffusion equation, Adv. Nonlinear Stud. 4 (2004) 251-272.

9. P. K. Maini, L. Malaguti, C. Marcelli and S. Matucci, Diffusion-aggregation processes with mono-stable reaction terms, Discr. Cont. Dyn. Syst. Ser. B 6 (2006) 1175-1189.

10. L. Malaguti and C. Marcelli, Sharp profiles in degenerate and doubly-degenerate Fisher-KPP equations, J. Diff. Eqns. 195 (2003) 471-496.

11. L. Malaguti, C. Marcelli and S. Matucci, A unifying approach to travelling wavefronts for reaction-diffusion equations arising from genetics and combustion models, Dyn. Syst. Appl. 12 (2003) 333-354.

12. J. D. Murray, Mathematical Biology, Vol. 1: An Introduction (Springer-Verlag, 2002).

13. V. Padrón, Effect of aggregation on population recovery modeled by a forwardbackward pseudoparabolic equation, Trans. Amer. Math. Soc. 356 (2004) 2739-2756.

14. F. Sánchez-Garduño and P. K. Maini, Travelling wave phenomena in some degenerate reaction-diffusion equations, J. Diff. Eqns. 117 (1995) 281-319.

15. P. Turchin, Population consequences of aggregative movements, J. Animal Ecol. 58 (1989) $75-100$. 